\title{
Arthroscopic retrieval of a large foreign body: A case report and literature review.
}

\author{
Yong Qin ${ }^{1}$, Ming Li², Songcen Lv ${ }^{1}$, Haile Pan ${ }^{*}$ \\ ${ }^{1}$ Orthopedic Surgery Section 4, the $2^{\text {nd }}$ Affiliated of Harbin Medical University, Harbin, PR China \\ ${ }^{2}$ Department of Sports Trauma and Arthroscopic Surgery, Zhengzhou Orthopaedics Hospital, Zhengzhou, PR China
}

\begin{abstract}
Background: We report the removal of a large metallic foreign body and review the relevant literature. Methods: A 28 y old woman was admitted with left knee swelling, pain, and limitation of activity resulting from an accident $7 \mathrm{~d}$ earlier. Radiography revealed a $3 \times 1 \mathrm{~cm}$ foreign body at the mid-tibial plateau. As the foreign body may have been metallic, it was not possible to perform magnetic resonance imaging to determine whether damage to the cruciate ligaments was present. Finally, the foreign body was retrieved.

Results: A $3 \times 4 \times 5 \mathrm{~cm}$ triangular broken knife blade was removed from the joint cavity. We excised the lateral meniscus, which had been damaged by the foreign body, and debrided the hyperplastic synovial membrane. Recovery was favorable with no complications. The patient was followed up at 6 months after surgery.

Conclusions: While foreign bodies in the knee are not uncommon, the presence of a large object is rare. When foreign bodies are small, most can be removed arthroscopically with a minimum of difficulty. In contrast, large foreign bodies within the knee joint cavity require an incision to open the joint for adequate exposure and removal of the object. Such a procedure can result in severe complications, such as infections and joint deformities. The decision to use one or the other approach requires careful consideration regarding the condition of the patient along with the skill, knowledge, and experience of the health care team.
\end{abstract}

Keywords: Metallic foreign body, X-ray, MRI.

Accepted on March 14, 2018

\section{Introduction}

The presence of a large foreign body within the knee is rare and is usually surgically managed. We report a case of a large Metallic Foreign Body (MFB) in the knee that was managed with arthroscopic retrieval. The patient was followed for 6 months with favorable results. The clinical manifestations, diagnosis, and treatment of a large MFB in the knee were discussed in this report and compared with those in the relevant literature.

\section{Case Report}

A 28 y old woman was admitted with left knee swelling, pain, and limitation of activity for one week due to an accident. No infection was present. On admission, her body temperature was $36.6^{\circ} \mathrm{C}$, with pulse $68 / \mathrm{min}$, respirations $20 / \mathrm{min}$, blood pressure $105 / 70 \mathrm{mmHg}$, normal development, and average nutritional status. A $3.5 \mathrm{~cm}$ laceration was present in the anterolateral left knee. Swelling was present, and a floating patella was noted. Laboratory results were unremarkable with no evidence of infection bacteriologic presence. Radiography revealed a $3 \times 1$ $\mathrm{cm}$ linear foreign body at the left tibial plateau. There was concern that the foreign body may have damaged the cruciate ligaments. As the patient indicated that the foreign body may be metallic, Magnetic Resonance Imaging (MRI) was not possible.

\section{Results}

The linear foreign body measured $3 \times 1 \mathrm{~cm}$ on a radiograph (Figure 1). Arthroscopy was performed to remove the object. The patella was approached inferolaterally, and a large collection of blood was found in the articular cavity, of which $10 \mathrm{ml}$ was saved for bacterial culture, and the articular cavity was then lavaged. Upon debridement of the synovial membrane, we found a large portion of a broken metal knife blade in the tibial plateau. Tissue was disrupted around the knife, and the femoral cartilage was damaged (Figure 2).

A fragment near the foreign body (Figure 3A) proved to be a portion of the anterior cruciate ligament (Figure 3B). After clearing this fragment, we found another fragment at the site where the knife was lodged in the tibial plateau (Figure 3C); this was also removed. 


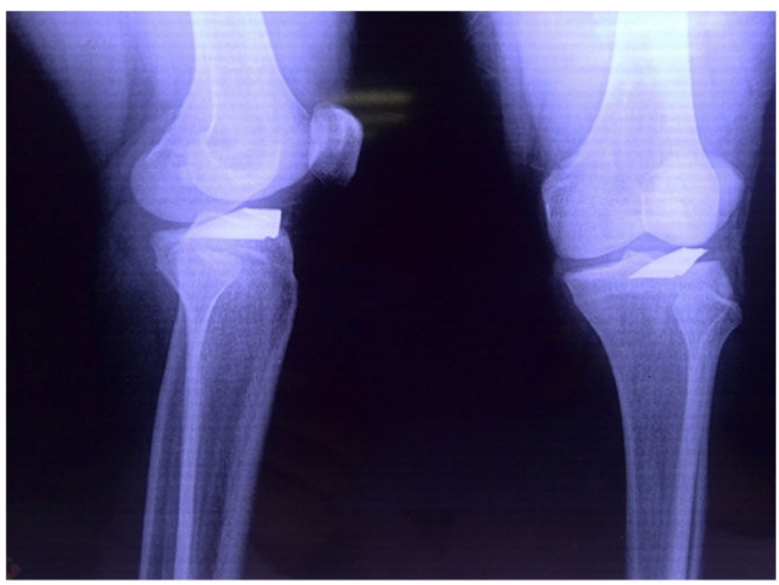

Figure 1. Radiograph of the left knee. A $3 \times 1 \mathrm{~cm}$ linear foreign object is lodged in the left tibial plateau.

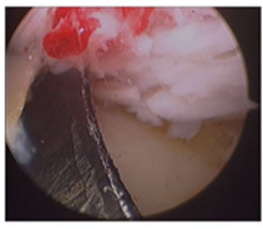

A

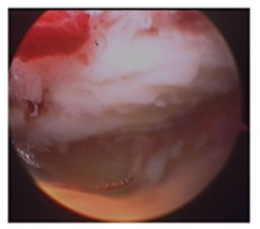

B

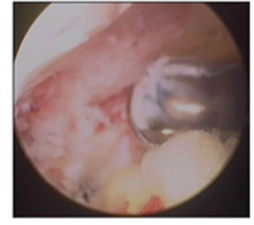

C
Figure 2. A. Large portion of a broken metal knife blade lodged in the tibial plateau. B. tissue disruption and C. cartilage damage around the foreign body.

The foreign body was inserted deep into the tibia (Figure 4A). Due to its smooth surface, the knife blade could not be removed easily (Figure 4B); the blade was broken and a portion had become displaced downward. We removed one piece while the remaining fragment remained in the knee joint (Figure 4C).

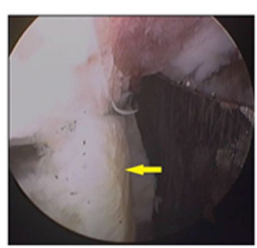

A

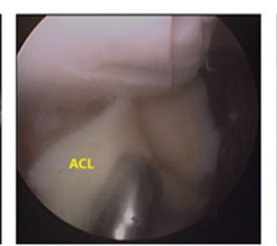

B

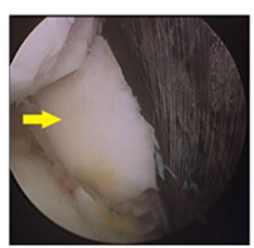

C
Figure 3. A fragment near the foreign body (A) proved to be part of the anterior cruciate ligament (B). ACL: Anterior Cruciate Ligament. Following removal of this fragment, another fragment was found $(C)$.

Numerous instruments were used in an attempt to dislodge the foreign body. A curette and pincers were used with an arthroscopic procedure to grasp the knife in the tibial plateau (Figures 5A and 5B). A rongeur was used to clean the bone around the knife and loosen the blade (Figure 5C).

Finally, we were able to remove a $3 \times 4 \times 5 \mathrm{~cm}$ triangular portion of the broken knife blade from the joint cavity. The knife was broken into 3 pieces (Figure 6A). The pieces were reconstructed to ensure the entire knife was removed. Examination under arthroscopy revealed that the lateral meniscus was transected, and its wedge shape was observed (Figure 6B). The medial meniscus was unaffected (Figure 6C). We excised the lateral meniscus and debrided the hyperplastic synovial membrane. Finally, the incision was sutured, and a drain tube inserted in the joint. The recovery was favorable with no complications, and the patient was followed up at 6 months following surgery.

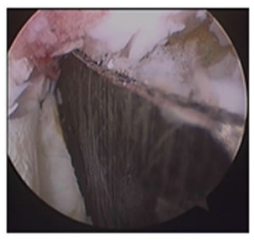

A

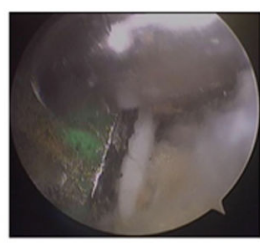

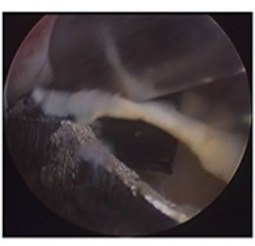

C
Figure 4. Location of the foreign body deeply lodged in the tibia (A). The surface of the knife was smooth (B). The knife blade was broken and a portion was displaced downward (C).

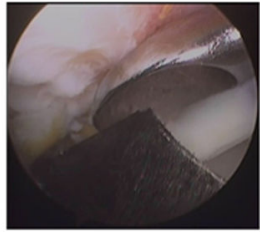

A

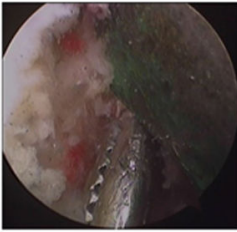

B

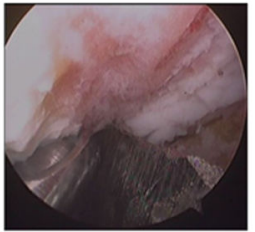

C
Figure 5. A number of surgical tools were used to dislodge the knife blade from the bone. The tools in the figure included a curette (A), pincers $(B)$ and a rongeur $(C)$.

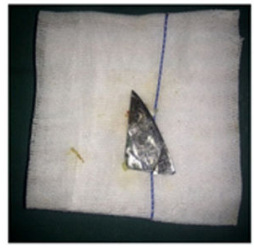

A

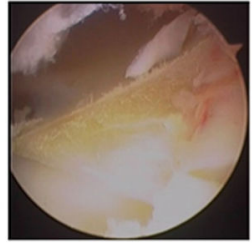

B

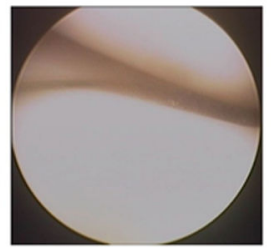

C
Figure 6. A. The broken knife. B. The wedge-shaped lateral meniscus. $C$. The medial meniscus was unaffected.

\section{Discussion}

While foreign bodies within the knee are not uncommon, the presence of a large foreign body is rare, with only a few reported cases. Yeung [1] and Oztekin [2] reported arthroscopic retrieval of sewing needle fragments from the knees of children. Lee [3] reported a rare case of an 8-year-old boy who accidentally fell, causing the point of a pencil to penetrate the left knee joint cavity. Devgan [4] reported an unusual injury involving a piece of glass that remained in the subcutaneous tissue for many years, eventually migrating into the knee joint cavity. Sharma [5] and Hafez [6] also reported cases in which glass was found within the knee joint. Palmers [7] reported an unusual metallic foreign body lodged in the lateral tibiofemoral compartment. Kim [8] used microarthroscopy to extract stainless steel particles embedded in the tibial plateau. Most case reports of foreign bodies in 
knee joints involve a bullet; 10 such reports exist in literature [9-18]. With magnification, it is relatively easy to extract small objects with arthroscopy. In contrast, large foreign bodies are difficult to dislodge completely from the knee joint. Such procedures should be performed by an experienced surgeon.

\section{Conclusions}

Small foreign bodies in the knee are not uncommon, while large foreign bodies are rare. Most small foreign bodies can be removed with arthroscopy. In particular, with magnification, it remains relatively easy to extract small objects with arthroscopy, thereby decreasing patient and surgeon concern about such procedures. Accordingly, we recommend arthroscopic retrieval for small objects. In contrast, arthroscopic retrieval of a large foreign body is difficult. As a result, large foreign objects within the knee joint cavity require an incision for exposure and removal of the object. Such surgery can result in severe complications, such as infections and joint deformities. Selecting the approach requires careful consideration of the condition of the patient and the skill, knowledge, and experience of the medical team.

\section{Declaration of Conflict of Interest}

None

\section{References}

1. Yeung Y, Wong JK, Yip DK, Kong JK. A broken sewing needle in the knee of a 4-year-old child: is it really inside the knee? Arthroscopy 2003; 19: 18-20.

2. Oztekin HH, Aslan C, Ulusal AE, Sertoz SZ, Ustaoglu RG, Fowler J. Arthroscopic retrieval of sewing needle fragments from the knees of 3 children. Am J Emerg Med 2006; 24: 506-508.

3. Lee BI, Choi HS, Kim JB, Min KD. Arthroscopic retrieval of a very rare penetrating foreign body of the knee. Arthroscopy 2004; 20: 1071-1074.

4. Devgan A, Mudgal KC. An unusual case of foreign body knee that spontaneously migrated inside and out of the joint: arthroscopic removal. Knee Surg Sports Traumatol Arthrosc 2007; 15: 758-760.

5. Sharma S, Rampurada A, Rees AJ. A glass foreign body in the knee joint mistaken for ACL avulsion: an unusual case. Knee Surg Sports Traumatol Arthrosc 2007; 15: 766-768.

6. Hafez MA, Al-Dars AM. Glass foreign bodies inside the knee joint following intra-articular injection. Am J Case Rep 2012; 13: 238-240.
7. Palmers M, Dierickx C, Peene P, Bijnens E. An unusual metallic foreign body in the lateral tibiofemoral compartment. Arthroscopy 2002; 18: 325-328.

8. Kim SJ, Lee YT, Kim HJ. Arthroscopic extraction of a stainless steel foreign body imbedded in the tibial plateau. Arthroscopy 1998; 14: 103-105.

9. Berg EE, Ciullo JV. Arthroscopic debridement after intraarticular low-velocity gunshot wounds. Arthroscopy 1993; 9: 576-579.

10. Cho MS, Warme WJ. Arthroscopic treatment of a transarticular low-velocity gunshot wound using tractoscopy. Arthroscopy 2002; 18: 532-537.

11. Hartford JM, Gorczyca JT. Late arthroscopic debridement of metal fragments and synovectomy after penetrating knee joint injury by low-velocity missile: a report of two cases. J Orthop Trauma 2001; 15: 222-224.

12. Gutierrez V, Radice F. Late bullet migration into the knee joint. Arthroscopy 2003; 19: 15.

13. Lee GH, Virkus WW, Kapotas JS. Arthroscopically assisted minimally invasive intraarticular bullet extraction: technique, indications, and results. J Trauma 2008; 64: 512-516.

14. Nikolic D, Vulovic R. Arthroscopy of the knee in war injuries. Injury 1996; 27: 175-176.

15. Petersen W, Beske C, Stein V, Laprell H. Arthroscopical removal of a projectile from the intra-articular cavity of the knee joint. Arch Orthop Trauma Surg 2002; 122: 235-236.

16. Sansone V, Mora L, de Spirito D. Arthroscopic retrieval of an unusual foreign body of the knee. Arthroscopy 2002; 18: 6.

17. Schroeder JE, Lowe J, Chaimsky G, Liebergall M, Mosheiff R. Migrating shrapnel: a rare cause of knee synovitis. Mil Med 2010; 175: 929-930.

18. White RR. Arthroscopic bullet retrieval. J Trauma 1987; 27: 455-456

\section{*Correspondence to}

Haile Pan

Orthopedic Surgery Section 4

The $2^{\text {nd }}$ Affiliated of Harbin Medical University

PR China 